\title{
Esofagitis aguda inducida por doxiciclina
}

\section{Doxycycline-induced acute esophagitis}

Autores: Raúl Real ${ }^{1}$, Daniel Cantero ${ }^{2}$, José Bellasai ${ }^{3}$

Artículo recibido: 2 enero 2014

Artículo aceptado: 16 febrero 2014

Resumen: se presenta caso de mujer joven con odinofagia intensa de inicio agudo. La endoscopía digestiva y biopsia esofágica confirman esofagitis isquémica aguda. Tras interrogatorio repetido se encontró asociación causal con ingesta previa de cápsula de doxiciclina utilizada para tratamiento de acné.

Palabras claves: esofagitis, doxiciclina, acné

Abstract: we present the case of a young woman with severe odynophagia of acute onset. The digestive endoscopy and esophageal biopsy confirmed an acute ischemic esophagitis. After repeated interviews, a causal association with prior ingestion of doxycycline capsule used for acne treatment was found.

Keywords: acute esophagitis, doxycycline, acne

Caso clínico: mujer de 24 años consulta por dolor a nivel retroesternal alto, de inicio súbito, que empeora con la deglución, inicialmente poco intenso pero que se acentúa con las horas, irradia a espalda. El dolor no cede con analgésicos comunes en gotas, se agrega disfagia que le impide la alimentación, tanto de sólidos como líquidos.

Antecedentes recientes: ingesta de $10 \mathrm{ml}$ de champagne horas antes. No ingesta de cáusticos en forma accidental ni provocada.

Antecedentes remotos: portadora de enfermedad celiaca de 10 años de evolución, en estricto tratamiento con dieta sin gluten, con mejoría sustancial de cuadro clínico inicial (dolor abdominal, pérdida de peso y anemia ferropénica). Actualmente no presenta deficiencias nutricionales, no pérdida de peso, sin trastornos tiroideos, hepáticos ni suprarrenales según chequeo del año anterior. Sus anticuerpos antitransglutaminasa y antiendomisio se mantienen persistentemente negativos en los últimos 5 años. No es portadora de hepatitis B ni HIV. No es etilista. Refiere pirosis ocasional y regurgitaciones ácidas muy ocasionales, sobre todo tras ingesta de café, que mejoran con omeprazol. Hábitos fisiológicos: normales

\footnotetext{
${ }^{1}$ Dpto. de Medicina Interna. Hospital Nacional. Itauguá, Paraguay.

${ }^{2}$ Endoscopía digestiva. Centro Médico La Costa. Asunción, Paraguay

${ }^{3}$ Profesor Emérito de la Facultad de Ciencias Médicas, U.N.A. Asunción, Paraguay
}

\section{Autor de correspondencia:}

Dr. Raúl Real

Jefe del Dpto. de Medicina Interna, Hospital Nacional. Itauguá. Paraguay

Tel (595)294321450

Correo electrónico: raulemilioreal@yahoo.com.ar 
Al examen físico no se constatan datos de valor. Sus signos vitales son normales. La faringe no está congestiva, no se observan aftas ni úlceras herpéticas.

Evolución: a las 48 hs del inicio del cuadro se la somete a endoscopía digestiva alta, hallándose esófago con calibre y distensibilidad conservadas. Mucosa de tercio superior, entre 25 y $30 \mathrm{~cm}$ de la arcada dental superior, con aspecto inflamatorio en forma circunferencial, con eritema leve y áreas de mucosa esfacelada, algo friable al contacto (fig 1 y 2). Transición a aproximadamente $38 \mathrm{~cm}$ de la arcada dentaria superior coincidente con el pinzamiento diafragmático. Mucosa de antro gástrico con eritema leve, de distribución difusa. Duodeno de aspecto normal. Se toman biopsias de esófago, estómago y duodeno. Se concluye como esofagitis difusa proximal y eritema antral leve. Dado que la paciente niega ingesta de cáusticos y el reflujo ácido gástrico es improbable que produzca lesiones sólo a nivel proximal, la etiología queda en duda.

\section{Figuras 1 y 2}

\section{Mucosa esofágica superior con eritema y áreas de mucosa esfacelada}

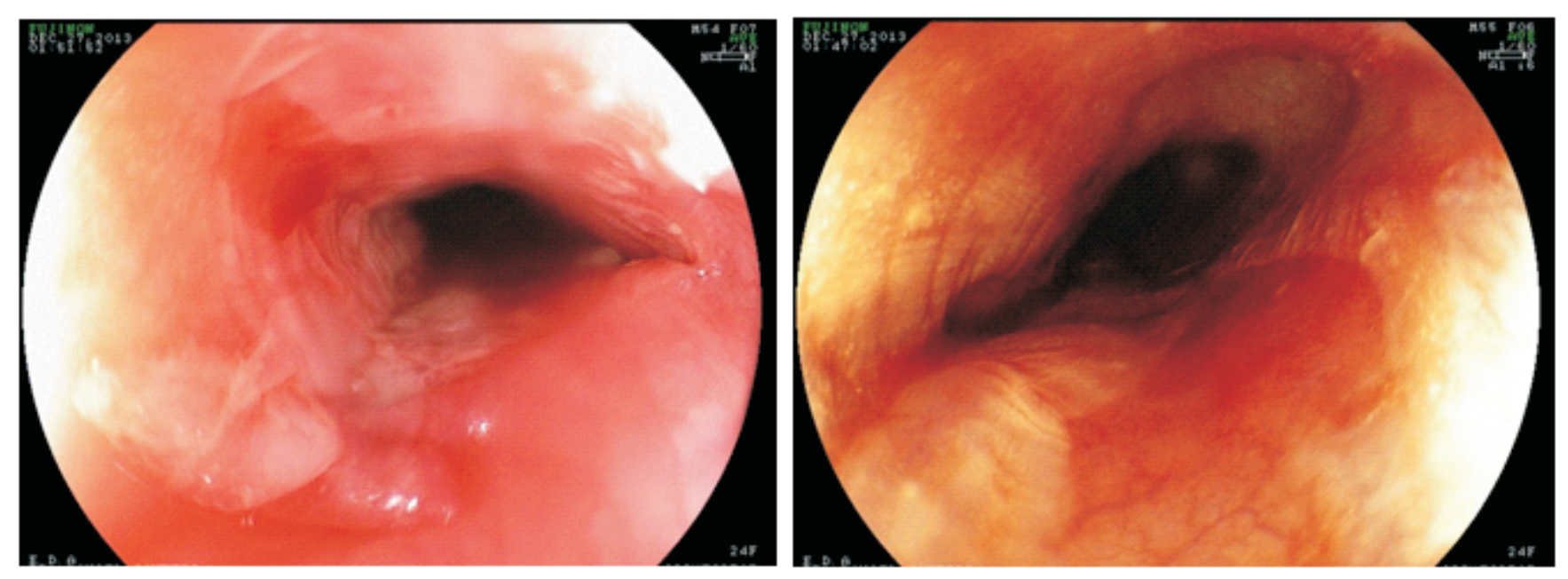

Es tratada con doble dosis de omeprazol, analgésicos en gotas (dipirona) y sucralfato, sin mejoría. A los tres días del inicio del cuadro se agregan mialgias y temperatura axilar $37,8^{\circ} \mathrm{C}$. No se descarta inicio de cuadro gripal de contagio intrafamiliar. Se solicita laboratorio por la posibilidad de una esofagitis de origen autoinmune o infecciosa.

Se obtiene hemograma con leve anemia normocítica normocrómica (hemoglobina 11,5 g/dL), leucocitos $9700 / \mathrm{mm}^{3}$, fórmula leucocitaria normal, eritrosedimentación de 13 y $24 \mathrm{~mm}$ a $1^{\circ}$ y $2^{\circ}$ hora respectivamente, plaquetas normales. La química sanguínea y hepatograma son normales. La proteína $\mathrm{C}$ reactiva está elevada $(6 \mathrm{mg} / \mathrm{dL})$. Los anticuerpos antinucleares y anti DNA son negativos, así como ANCA c y ANCA p. Los anticuerpos contra hepatitis B, HIV, citomegalovirus y herpes simple tipo 1 son todos negativos. Los marcadores de enfermedad celiaca antitransglutaminasa IgA y antiendomisio IgA se hallan también negativos.

La fiebre no se repite en los días siguientes pero la disfagia es intensa y la paciente se deshidrata levemente por lo que requiere internación de 24 hs para hidratación parenteral. Anatomopatólogo informa esofagitis erosiva ulcerativa aguda con llamativa necrosis de tipo isquémico, de origen difícil de determinar (fig 3). La mucosa gástrica no presenta alteraciones patológicas, no Helicobacter pylori. La mucosa duodenal presenta subatrofia vellositaria leve. 


\section{Figura 3}

Mucosa esofágica, zona de borde de úlcera. Nótese núcleos de coloración basófila difusa, en apostosis (necrosis) y denso infiltrado inflamatorio agudo que se extiende por debajo de la mucosa. H.E. 20x

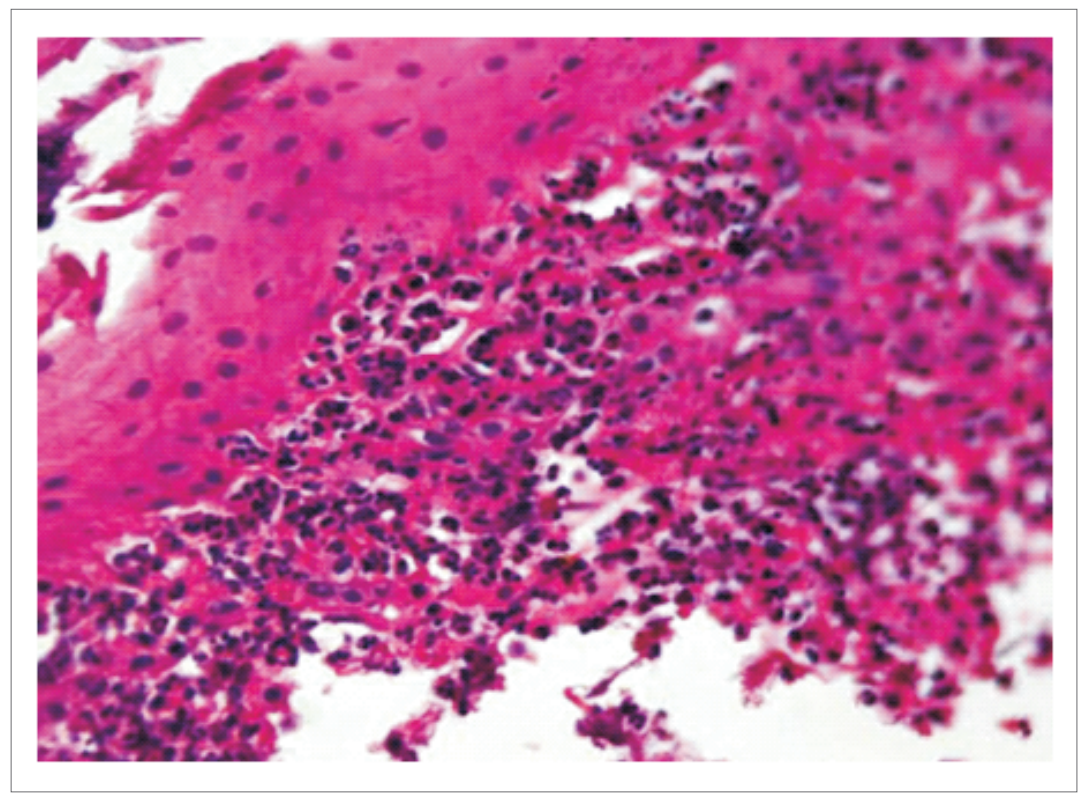

Al sexto día del inicio la enfermedad, ante la persistencia del cuadro, la pérdida de $3 \mathrm{~kg}$ de peso y sin hallazgos laboratoriales orientadores del diagnóstico se plantea otra endoscopía digestiva alta y colocación de sonda nasogástrica para alimentación enteral. Pero reinterrogando a la paciente, ésta admite haber ingerido una cápsula de doxiciclina la noche antes del inicio de su afección actual, al acostarse y aparentemente con escaso líquido. Dicho medicamento fue indicado por Dermatólogo para tratamiento cíclico de acné crónico. La forma farmacéutica en cápsulas fue una elaboración magistral para evitar el contenido de gluten entre los excipientes.

Se asume en ese momento una esofagitis medicamentosa y se expecta la segunda endoscopía. Al octavo día de enfermedad la ingesta de líquidos mejora y la disfagia disminuye paulatinamente. Los síntomas ceden al $11^{\circ}$ día, quedando sin secuelas.

\section{Discusión}

El inicio súbito de odinofagia acompañada de disfagia hace suponer clínicamente un cuadro de esofagitis, diagnóstico que rápidamente confirmado en esta paciente con una endoscopía digestiva alta. ${ }^{1}$ Lo llamativo del caso fue la etiología debido a que inicialmente no había antecedentes patológicos que orientaran a la causa de la lesión esofágica. La ingesta de unos ml de bebida alcohólica no justificaba tanta inflamación.

Las esofagitis es una afección de múltiples etiologías. ${ }^{2}$ La cáustica es la primera en tenerse en cuenta por la localización alta observada en esta paciente, pero, llamativamente, no había lesión labial o en la orofaringe ni antecedente de ingesta, accidental ni provocada, de sustancias irritantes ácidas ni alcalinas.

Otras causas tenidas en cuenta fueron las infecciones por bacterias, hongos, virus o parásitos. ${ }^{3}$ La negatividad de la serología IgM contra citomegalovirus y herpes simple tipo 1 descartó estas infecciones. La inmunocompetencia comprobada en la paciente alejó la posibilidad de otros agentes infecciosos oportunistas. La candidiasis era poco probable por el aspecto macroscópico observado por el endoscopista, además por el hecho de no ser una paciente alcohólica ni desnutrida. La enfermedad por reflujo gastroesofágico pareció una causa con escaso sustento pues no había incompetencia cardial ni lesión mucosa del tercio inferior del esófago. 
Ante la posibilidad de esofagitis eosinofílica, dermatitis bullosa y vasculitis se realizaron biopsias. Otra posibilidad diagnóstica tenida en cuenta fue la Enfermedad inflamatoria intestinal pues existen casos de Enfermedad de Crohn esofágica. ${ }^{4,5}$ Esta puede tener un inicio agudo, no tiene marcadores inmunológicos específicos y las lesiones pueden no observarse en una biopsia superficial como la efectuada en las esofagitis. ${ }^{6,7}$ La Enfermedad inflamatoria intestinal se puede asociar a la Enfermedad celiaca, pues ambas son autoinmunes. ${ }^{8-10}$

Sólo con el reinterrogatorio se pudo obtener, días después, el antecedente de la ingesta del antibiótico la noche previa al comienzo de sus molestias, por lo que se asumió como una esofagitis por doxiciclina. Los medicamentos más frecuentemente implicados en la esofagitis medicamentosa son las tetraciclinas (como la doxiciclina), otros antibióticos (ampicilina, amoxicilina), bifosfonatos, quinidina, antiinflamatorios no esteroideos (sobre todo el ác. acetilsalisílico), corticoides, ác. ascórbico, sulfato ferroso, cloruro potásico, antirretrovirales, teofilina y lanzoprazol. ${ }^{3,11}$ Se localiza con mayor frecuencia en el esófago medio o superior, coincidiendo con el cayado aórtico. No siempre existe un obstáculo anatómico ni alteración motora en la deglución que favorezca esta afección. Los factores de riesgo se relacionan al paciente y al fármaco (ver tabla 1). ${ }^{1,12,13}$ Histológicamente, los hallazgos son inespecíficos: necrosis y exudados inflamatorios de linfocitos y eosinófilos. Se ha demostrado que la doxiciclina se acumula en la capa basal del epitelio esofágico y desencadena toxicidad celular inhibiendo la síntesis de proteínas, el recambio y reparación celular. Algunos autores han descrito alteraciones sugestivas de vasculitis. ${ }^{14}$ La sintomatología tarda 8 a 10 días en ceder. ${ }^{13,15,16}$

\section{TABLA 1}

Factores de riesgo para la esofagitis medicamentosa

Factor es relacionados al paciente

Posición supina luego de la deglución Ingesta insu ficiente de fluidos

Disminución en la producción de saliva Contacto pro longado con la mucosa Estrecheces anatómicas o dismotilidad
Factores relacionados al fármaco

Concentración del fármaco

$\mathrm{pH}$ del fármaco

Medicamentos de liberación prolongada

Presentación en forma de cápsulas

Tamaño y solubilidad del fármaco

La paciente fue tratada desde el inicio con omeprazol a doble dosis, sucralfato y analgésicos pues fue tipificada como una esofagitis cáustica grado II a (ver tabla 2). ${ }^{2}$ No existe un tratamiento específico para la esofagitis medicamentosa y es inusual la aparición de secuelas. ${ }^{13,15}$

\section{TABLA 2}

\section{Clasificación de la esofagitis cáustica*}

\begin{tabular}{|ll|}
\hline Tipo I & eritema y edema de mucosa \\
\hline Tipo II a & erosiones y úlceras superficiales \\
\hline Tipo II b & erosiones y úlceras profundas \\
\hline Tipo III a & $\begin{array}{l}\text { pequeñas áreas de necrosis con zonas de mucosa } \\
\text { más decolorada, con aspecto gris o marrón }\end{array}$ \\
\hline Tipo III b & extensas áreas de necrosis \\
\hline Tipo IV & perforación \\
\hline
\end{tabular}

*Argüelles Arias F et al. Medicine. 2008; 10(1): 12-9 
Afortunadamente la recuperación de esta paciente fue total. La frecuencia con que aparecen estenosis esofágicas post ingesta de cáusticos es mayor: oscila entre 15 y 35\% y se relaciona con el grado de lesión inducida por el agente ingerido. Pueden hacerse sintomáticas hacia la segunda o tercera semanas después de una fase latente de reparación o, en otros casos, meses o años después de la ingesta. ${ }^{17}$

La doxiciclina es de uso común en el tratamiento del acné y es frecuente encontrar reportes de este efecto colateral relacionado al uso dermatológico. ${ }^{12}$ Los propósitos de este reporte son recalcar a los médicos la necesidad de insistir con los pacientes en la ingesta adecuada de sus medicamentos y destacar el valor de la anamnesis, que en este caso hubiera hecho el diagnóstico precozmente. ${ }^{13}$

\section{Referencias bibliográficas}

1. Martínez Crespo JJ, Martínez de Guzmán M. Esophagitis due to drugs: unusual aetiology of dysphagia. Farm Hosp. 2005 Jan-Feb; 29(1): 71-2.

2. Argüelles Arias F, Sánchez-Gey Venegas S, Herrerías Gutiérrez JM. Otras esofagitis. Etiología infecciosa. Etiología tóxica/cáustica. Esofagitis eosinofílica Medicine. 2008; 10(1): 12-9

3. Pociello Almiñana N, Vilar Escrigas P, Luaces Cubells C. Doxycycline-induced esophagitis: report of two cases. An Pediatr (Barc). 2005 Feb; 62(2): 171-3.

4. Laass MW, Roggenbuck D, Conrad K. Diagnosis and classification of Crohn's disease. Autoimmun Rev. 2014 Jan 11. pii: S1568-9972(14)00041-X.

5. Feagans J, Victor D, Joshi V. Crohn disease of the esophagus: a review of the literature. South Med J. 2008 Sep; 101(9): 927-30.

6. Pantanowitz L, Gelrud A, Apstein M, Nasser I. Crohn's disease of the esophagus. Ear Nose Throat J. 2004 Jun; 83(6): 422-3.

7. Decker GA, Loftus EV Jr, Pasha TM, Tremaine WJ, Sandborn WJ. Crohn's disease of the esophagus: clinical features and outcomes. Inflamm Bowel Dis. 2001 May; 7(2): 113-9.

8. Ouaka-Kchaou A, Ennaifer R, Elloumi H, Gargouri D, Hefaiedh R, Kochlef A et al. Autoimmune diseases in coeliac disease: effect of gluten exposure. Therap Adv Gastroenterol. 2008 Nov; 1(3): 169-72.

9. Baum S, Sakka N, Artsi O, Trau H, Barzilai A. Diagnosis and classification of autoimmune blistering diseases. Autoimmun Rev. 2014 Jan 13. pii: S1568-9972(14)00059-7.

10. Zhernakova A, Withoff S, Wijmenga C. Clinical implications of shared genetics and pathogenesis in autoimmune diseases. Nat Rev Endocrinol. 2013 Nov; 9(11): 646-59.

11. Al Mofarreh MA, Al Mofleh IA. Doxycline-induced esophageal ulcerations. Saudi J Gastroenterol. 1998 Jan; 4(1): 20-4.

12. Guttman OR, Zachos M. Drug-induced esophageal injury with an occult vascular ring. Paediatr Child Health. 2011 Nov; 16(9): 554-6.

13. Vãlean S, Petrescu M, Cãtinean A, Chira R, Mircea PA. Pill esophagitis. Rom J Gastroenterol. 2005 Jun; 14(2): 159-63.

14. Medlicott SA, Ma M, Misra T, Dupre MP. Vascular wall degeneration in doxycycline-related esophagitis. Am J Surg Pathol. 2013 Jul; 37(7): 1114-5.

15. Al Mofarreh MA, Al Mofleh IA. Esophageal ulceration complicating doxycycline therapy. World J Gastroenterol. 2003 Mar; 9(3): 609-11.

16. Kadayifci A, Gulsen MT, Koruk M, Savas MC. Doxycycline-induced pill esophagitis. Dis Esophagus. 2004;17(2):168-71.

17. Cuenca Torres O, Ramírez Sotomayor J, Martínez Arguello D. Estenosis esofágica por ingestión de cáusticos. Rev. Nac. (Itauguá) 2012; 4 (1): 53-54 